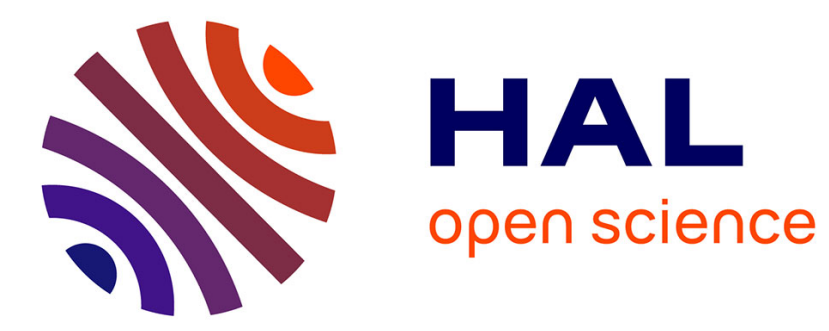

\title{
Chemical reaction for mixing studies
}

Emilie Guilbert, Christophe Almarcha, Emmanuel Villermaux

\section{To cite this version:}

Emilie Guilbert, Christophe Almarcha, Emmanuel Villermaux. Chemical reaction for mixing studies. Physical Review Fluids, 2021, 6 (11), pp.114501. 10.1103/physrevfluids.6.114501 . hal-03521836

\section{HAL Id: hal-03521836 https://hal.science/hal-03521836}

Submitted on 11 Jan 2022

HAL is a multi-disciplinary open access archive for the deposit and dissemination of scientific research documents, whether they are published or not. The documents may come from teaching and research institutions in France or abroad, or from public or private research centers.
L'archive ouverte pluridisciplinaire HAL, est destinée au dépôt et à la diffusion de documents scientifiques de niveau recherche, publiés ou non, émanant des établissements d'enseignement et de recherche français ou étrangers, des laboratoires publics ou privés. 


\title{
Chemical reaction for mixing studies
}

\author{
Emilie Guilbert $\odot,{ }^{1}$ Christophe Almarcha $\odot,{ }^{1}$ and Emmanuel Villermaux $\odot^{1,2, *}$ \\ ${ }^{1}$ Aix Marseille Université, CNRS, Centrale Marseille, IRPHE UMR 7342, 13384 Marseille, France \\ ${ }^{2}$ Institut Universitaire de France, 75005 Paris, France
}

(Received 22 December 2020; accepted 27 October 2021; published 16 November 2021)

We introduce an original chemical reaction between two transparent reactant solutions (fluorescin and potassium ferricyanide), producing a fluorescent product in water (fluorescein). The reaction has a tunable kinetics, allowing the quantitative investigation of the interplay between molecular diffusion and reaction kinetics in various reactant field topologies. We document the chemical reaction kinetics and its sensitivity to ambient $\mathrm{pH}$, temperature, reactants concentrations, etc., and we implement it in two simple reactant-contacting geometries in a Hele-Shaw cell. The resulting reaction-diffusion zones and product formation rates exhibit either the reaction-controlled or diffusion-controlled regimes, as well as a new "diffusio-chemical" regime which we show to be inherent to the early time transient dynamics of any bimolecular reaction between initially segregated reactants. The potential interest of this reaction, opening prospects for the study of reactive mixing, is underlined.

DOI: 10.1103/PhysRevFluids.6.114501

\section{INTRODUCTION}

The very notion of chemical reaction between different species contains in itself the notion of mixing: For a chemical reaction between initially separated species to occur, these must be first mixed at the molecular level. This last but one step, that is, the interpenetration of the phases present before they react, takes some time and may compete with the chemical transformation itself [1]. A number quantifies this competition: If $t_{c}$ is the time required for chemical conversion between ideally mixed reactants [2], and $t_{s}$ is the time it takes for the species to mix at the molecular level, Damköhler [3] singled out that it is when the ratio

$$
\mathrm{Da}=\frac{t_{s}}{t_{c}}
$$

is larger than unity that the problem of estimating the global conversion rate of the reactants shifts from a pure chemical kinetics problem to a problem involving both chemical kinetics and the way the medium where the reaction takes place is stirred, then mixed (see Ref. [4] for the distinction between these two notions and the notations).

To address this problem experimentally, it is desirable to have a way to make the reactants and/or the products of the reaction measurable locally quantitatively, and ideally fluorescent in order to exploit laser-induced fluorescence methods. Clock reactions involving iodine [5], and some other consecutive or parallel reactions like azo-coupling reactions [6,7] partially achieve this goal, have a relatively tunable chemical time $t_{c}$, but involve several intermediate steps which make it difficult to decipher the impact of molecular mixing on the overall production rate of the reaction outcome.

*emmanuel.villermaux@univ-amu.fr 


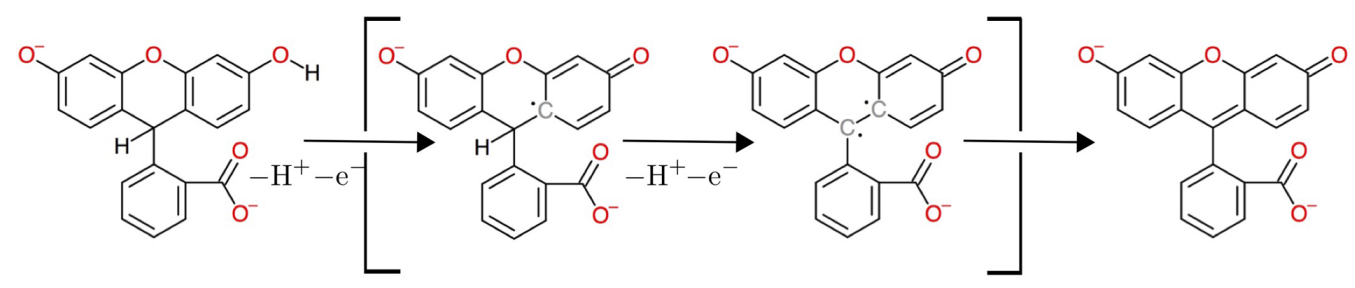

FIG. 1. Skeletal formulas of fluorescin (left) and fluorescein (right). The overall oxidation mechanism of fluorescin involves two successive losses of protons $\mathrm{H}^{+}$.

Fluorescent dyes like fluorescein are extremely convenient chemical tracers due to their sensitivity to the acidity of the medium, like $\mathrm{pH}$ indicators [8]. Fluorescein in its acidic form does not fluoresce, whereas it does in its basic form (for $\mathrm{pH}>4-5$ ). This method has been used in many instances with elementary stirring protocols like vortices $[9,10]$ or more complicated ones like in turbulent jets $[11,12]$. However, the acido-basic neutralization is an extremely fast reaction $\left(t_{c} \approx 10^{-10} \mathrm{~s}\right.$, see Refs. $\left.[13,14]\right)$, which corresponds, in most situations, to the singular $\mathrm{Da} \rightarrow \infty$ limit. The use of fluorescein thus serves more as a tool to trace iso-pH surfaces than for studying the interplay between reaction and mixing per-se [15]. The same remark applies to other fast reactions [7,16-18].

There is, however, a constant need to have at one's disposal a chemical signal, produced via a proper chemical reaction, reflecting the intrinsic dynamics of any system having its own kinetics, may it be biological [19-21] or not [22,23].

The dynamics of reaction-diffusion systems on still substrates has been the subject of considerable attention: The irreversible bimolecular reaction $\mathrm{A}+\mathrm{B} \rightarrow \mathrm{C}$ between two initially separated reactants $\mathrm{A}$ and $\mathrm{B}$ producing an inert product $\mathrm{C}$ has been studied either for interdiffusing species [24,25], for one species being static [26], for different initial concentrations of the reactants and/or relative diffusivities of the two reacting species [27-29], for example. In all cases, mostly concerned with the large-Da limit, the emphasis has been placed at describing the temporal dependence of the reaction rate, of the reaction zone width, of its location in space, and of the net amount of product formed. However, very few of these predictions have been tested experimentally (see nevertheless Refs. $[28,29]$ ), precisely due to the lack of a proper chemical reaction to diagnose the system, and address them.

Here, we introduce a chemical reaction inspired by forensic studies [30] involving transparent reactants, which produces a fluorescent product (fluorescein), with a chemical time $t_{c}$ adjustable over several orders of magnitude. We first document the chemical reaction itself in Sec. II where we detail its kinetics and then implement it in two different configurations where reaction competes with molecular diffusion in Sec. III. These experiments validate the performance of this reaction as a diagnostic tool and demonstrate its ability to highlight different reaction-diffusion regimes in simple configurations.

\section{THE REACTION}

The reaction is the oxidation-reduction transforming fluorescin dianion $\mathrm{C}_{20} \mathrm{H}_{12} \mathrm{O}_{5}^{2-}$ into fluorescein dianion $\mathrm{C}_{20} \mathrm{H}_{10} \mathrm{O}_{5}^{2-}$. This irreversible reaction takes place between fluorescin and potassium ferricyanide to produce fluorescein which fluoresces in aqueous solution [see (2) and Fig. 1]:

$$
\mathrm{C}_{20} \mathrm{H}_{12} \mathrm{O}_{5}^{2-}+2 \mathrm{OH}^{-}+2 \mathrm{Fe}^{3+} \rightarrow \mathrm{C}_{20} \mathrm{H}_{10} \mathrm{O}_{5}^{2-}+2 \mathrm{Fe}^{2+}+2 \mathrm{H}_{2} \mathrm{O} .
$$

The reaction mechanism, preparation protocol of fluorescin and the features of the chemical kinetics of this reaction are as follows: 
TABLE I. Redox potentials.

\begin{tabular}{lcc}
\hline \hline Oxidizing agent & Redox potentials (V) & Reducing agent \\
\hline $\mathrm{Fe}^{3+}$ & 0.77 & $\mathrm{Fe}^{2+}$ \\
$\mathrm{C}_{20} \mathrm{H}_{10} \mathrm{O}_{5}^{2-}$ & 0.66 & $\mathrm{C}_{20} \mathrm{H}_{11} \mathrm{O}_{5}^{2-}$ \\
$\mathrm{C}_{20} \mathrm{H}_{11} \mathrm{O}_{5}^{2-}$ & 0.33 & $\mathrm{C}_{20} \mathrm{H}_{12} \mathrm{O}_{5}^{2-}$ \\
\hline \hline
\end{tabular}

\section{Mechanism}

The oxidation mechanism of the reaction is of the Gif-Fenton [31] type which occurs in two steps, each corresponding to a simultaneous loss of a proton $\mathrm{H}^{+}$and of an electron with the formation of a radical intermediate, as shown in Fig. 1. The reduction of fluorescein into fluorescin involves the same mechanism but reversed: the two steps correspond to the gain of a proton $\mathrm{H}^{+}$and an electron which result in breaking the double bond responsible for the fluorescence of fluorescein. To oxidize fluorescin we choose to use a potassium ferricyanide solution. Potassium ferricyanide dissociates in water [see (3) and (4)] to form $\mathrm{Fe}^{3+}$ ions which reacts with fluorescin as

$$
\begin{gathered}
\mathrm{K}_{3} \mathrm{Fe}(\mathrm{CN})_{6} \rightarrow 3 \mathrm{~K}^{+}+\mathrm{Fe}(\mathrm{CN})_{6}^{3-}, \\
\mathrm{Fe}(\mathrm{CN})_{6}^{3-} \rightarrow \mathrm{Fe}^{3+}+6 \mathrm{CN}^{-} .
\end{gathered}
$$

The redox couples involved in this reaction are $\mathrm{Fe}^{3+} / \mathrm{Fe}^{2+}$ and $\mathrm{C}_{20} \mathrm{H}_{12} \mathrm{O}_{5}^{2-} / \mathrm{C}_{20} \mathrm{H}_{10} \mathrm{O}_{5}^{2-}$, each associated with the half-equations (5) and (6):

$$
\begin{gathered}
\mathrm{Fe}^{3+}+e^{-} \rightarrow \mathrm{Fe}^{2+} \\
\mathrm{C}_{20} \mathrm{H}_{10} \mathrm{O}_{5}^{2-}+2 e^{-}+2 \mathrm{H}_{2} \mathrm{O} \rightarrow \mathrm{C}_{20} \mathrm{H}_{12} \mathrm{O}_{5}^{2-}+2 \mathrm{OH}^{-} .
\end{gathered}
$$

The redox potential of the couple $\mathrm{Fe}^{3+} / \mathrm{Fe}^{2+}$, well documented in the literature [32], is $0.77 \mathrm{~V}$; the redox potential of the second couple is, however, unknown. We have performed specifically designed cyclic voltammetry measurements of the fluorescin solution [33], which highlight two successive oxidations corresponding to the two steps of the oxidation mechanism illustrated in Fig. 1. The first oxidation occurs at $0.33 \mathrm{~V}$ while the second oxidation, which takes place at $0.66 \mathrm{~V}$, corresponds to the appearance of fluorescence. The redox potentials are summarized in Table I and confirm that fluorescin is oxidized by the ions $\mathrm{Fe}^{3+}$ since the potential of the couple $\mathrm{Fe}^{3+} / \mathrm{Fe}^{2+}$ is larger than the two potentials of the couple $\mathrm{C}_{20} \mathrm{H}_{12} \mathrm{O}_{5}^{2-} / \mathrm{C}_{20} \mathrm{H}_{10} \mathrm{O}_{5}^{2-}$.

\section{Preparation of fluorescin}

Fluorescine (A, see the nomenclature in Table II) is used in the forensic context to detect traces of blood, which contains iron, to produce fluorescein (F) according to (2). A can be prepared from $\mathrm{F}$ itself: the reduction of $\mathrm{F}$ to $\mathrm{A}$ is made from zinc in powder and sodium hydroxide in a distilled

TABLE II. Abbreviation nomenclature of the chemical species, and their diffusion coefficients in water.

\begin{tabular}{lccc}
\hline \hline Name & Formula & Symbol & Diffusion coefficient $D_{i}\left(\mathrm{~m}^{2} \mathrm{~s}^{-1}\right)$ \\
\hline Fluorescin & $\mathrm{C}_{20} \mathrm{H}_{12} \mathrm{O}_{5}^{2-}$ & $\mathrm{A}, c_{A}$ & $1.3 \times 10^{-10}$ \\
Potassium ferricyanide & $\mathrm{K}_{3} \mathrm{Fe}(\mathrm{CN})_{6}$ & $\mathrm{~B}, c_{B}$ & $5.4 \times 10^{-10}$ \\
Fluorescein & $\mathrm{C}_{20} \mathrm{H}_{10} \mathrm{O}_{5}^{2-}$ & $\mathrm{F}, c$ & $1.3 \times 10^{-10}$ \\
Rhodamine & $\mathrm{C}_{28} \mathrm{H}_{31} \mathrm{~N}_{2} \mathrm{O}_{3} \mathrm{Cl}$ & $\mathrm{Rh}, c_{\mathrm{Rh}}$ & $4.1 \times 10^{-10}$ \\
\hline \hline
\end{tabular}


water solution as

$$
\mathrm{C}_{20} \mathrm{H}_{10} \mathrm{O}_{5}^{2-}+2 \mathrm{H}_{2} \mathrm{O}+\mathrm{Zn} \leftrightarrow \mathrm{C}_{20} \mathrm{H}_{12} \mathrm{O}_{5}^{2-}+2 \mathrm{OH}^{-}+\mathrm{Zn}^{2+} .
$$

$\mathrm{F}$ is usually available as a powder (fluorescein disodium salt), $0.25 \mathrm{~g}$ of which are dissolved into a flask filled with $50 \mathrm{ml}$ of distilled water to produce a reddish brown solution which, after the addition of $7.5 \mathrm{~g}$ of $\mathrm{Zn}$ powder and $3 \mathrm{~g}$ of $\mathrm{NaOH}$ pellets is stirred for 1 minute until the $\mathrm{NaOH}$ pellets have dissolved. The reaction being moderately exothermic, heating of the flask may be noticed. The flask must be airtight and protected from light (e.g., coated with aluminium foil). Then, stirring is stopped to allow for the sedimentation of the $\mathrm{Zn}$ particles for about 30 minutes, after which a transparent solution of $\mathrm{A}$ is obtained, with a layer of $\mathrm{Zn}$ sediments at the bottom of the flask. The A solution can remain transparent, colorless, and nonfluorescent for weeks if stored in a dark, oxygen-free environment and remains in contact with the $\mathrm{Zn}$ powder. We have typically used the solution not more than 20 minutes after preparation.

\section{Reaction chemical kinetics and apparent order}

For the sake of brevity, we assign letters to the different species according to Table II and we note $c_{i}$ and $D_{i}$ the molar concentration of species $i$, and its diffusion coefficient in water, respectively. The method for determining the diffusion coefficient $D_{B}$ of potassium ferricyanide is explained in the Appendix. Rhodamine is not involved in the reaction but is a useful tracer.

To study the dependencies of the reaction rate of (2), which coincides with the production rate $\dot{c}$ of $\mathrm{F}$, to the reactants $\mathrm{A}$ and $\mathrm{B}$ concentration, medium $\mathrm{pH}$ and temperature, we use a traditional method consisting in premixing the reactants in a vigorously stirred tank (so that mixing is not limiting) and measuring the production rate of $\mathrm{F}$, taking advantage of its fluorescence: A $488 \mathrm{~nm}$ laser sheet shines through a $5 \mathrm{~cm}^{3}$ tank filled with $\mathrm{B}$ placed on a magnetic stirrer (spinning at $f=10 \mathrm{~Hz}$ ) and a camera records the increasing fluorescence signal, proportional to $c$ [Figs. 2(a) and 2(b)], uniform in the tank volume, thanks to efficient mixing. Typical F production times $t_{c}$ are of order $10-100 \mathrm{~s}$, so that $\mathrm{Da}=1 /\left(f t_{c}\right)=O\left(10^{-2}\right)-O\left(10^{-3}\right)$, indeed smaller than unity.

To determine the partial orders of the reaction, we proceed with two measurements: one with the reactants at stoichiometry [i.e., $c_{A 0}=c_{B 0} / 2$, see Fig. 2(b), right] and another with one of the reactants introduced in excess [i.e., $c_{B 0} \gg c_{A 0}$, see Fig. 2(b), left]. In both limits, the observed kinetics is consistent with partial orders with respect to reactants $\mathrm{A}$ and $\mathrm{B}$ equal to unity, corresponding to a reaction of total order two, namely,

$$
\dot{c}=k c_{A} c_{B}
$$

leading, for the two initial concentration-ratio limits mentioned above, to

$$
\begin{gathered}
1-c / c_{A 0}=\frac{1}{1+t / t_{c}}, \text { at stoichiometry for } c_{A 0}=c_{B 0} / 2, \\
1-c / c_{A 0}=e^{-t / t_{c}}, \text { when } c_{B 0} \gg c_{A 0}, \\
\text { with } t_{c}=\left(k c_{B 0}\right)^{-1} .
\end{gathered}
$$

This fact can be understood from a detailed balance involving the intermediate radical $\mathrm{X}$ sketched in Fig. 1 and noting that the two-stage oxidation process can be written as

$$
\begin{aligned}
& \mathrm{A}+\mathrm{B} \stackrel{k}{\longrightarrow} \mathrm{X}, \\
& \mathrm{X}+\mathrm{B} \stackrel{k_{1}}{\longrightarrow} \mathrm{F},
\end{aligned}
$$

for a global balance $\mathrm{A}+2 \mathrm{~B} \rightarrow \mathrm{F}$. A steady-state approximation for the intermediate species $\mathrm{X}$ leads to $c_{\mathrm{X}}=\frac{k}{k_{1}} c_{A}$, showing that, since $\dot{c}=k_{1} c_{\mathrm{X}} c_{B}$ from the second balance equation above, the overall kinetics is of order unity with respect to $\mathrm{A}$ and $\mathrm{B}$, as in (7). 

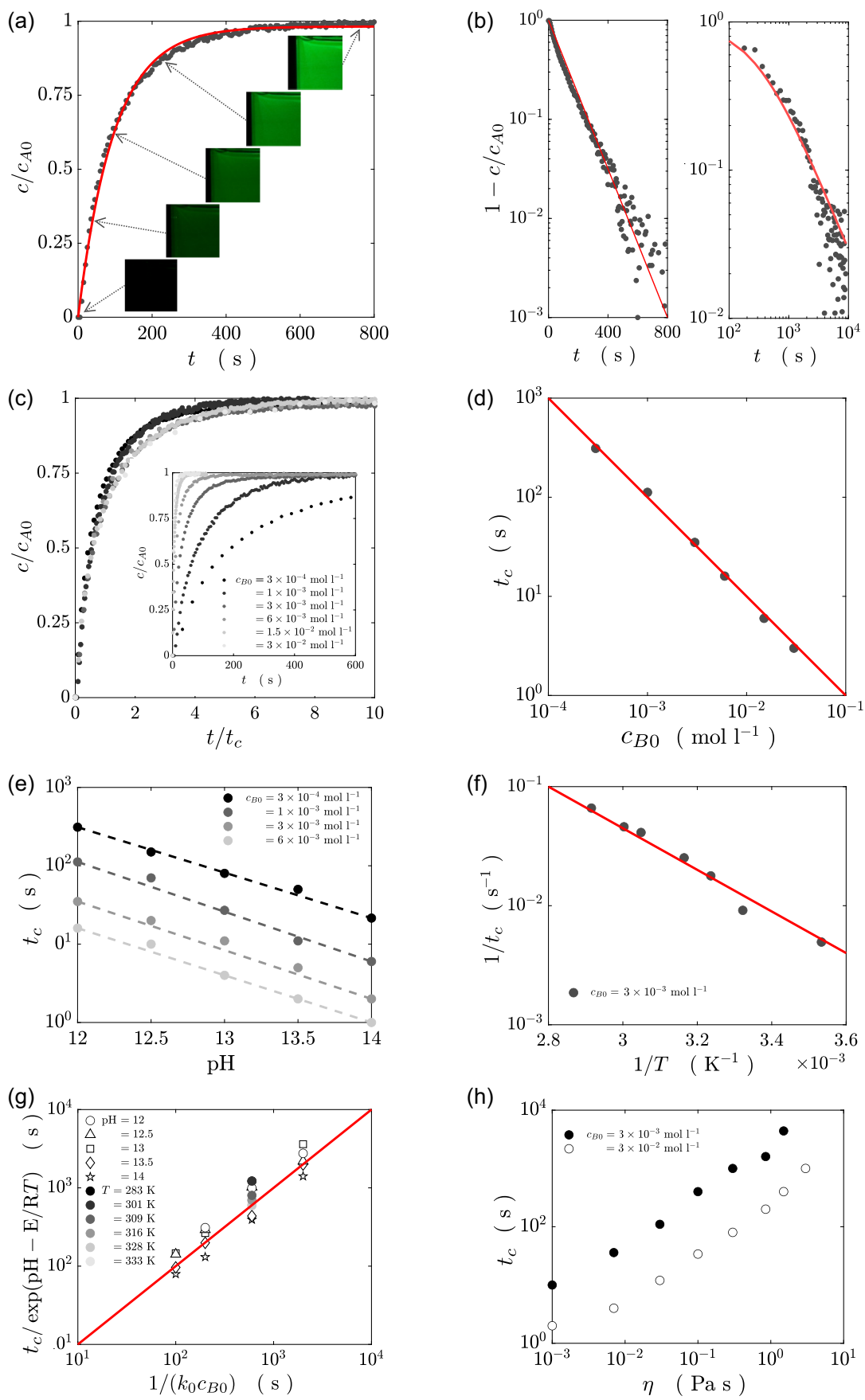

FIG. 2. Reaction kinetics. (a) Temporal increase of the fluorescent product $\mathrm{F}$ concentration in the mixing box, and fit by a first-order exponential relaxation $\left(c_{A 0}=3 \times 10^{-5} \mathrm{moll}^{-1}\right)$ in the case $c_{B 0} \gg c_{A 0}$. (b) (left) representation in semilogarithmic units when $c_{B 0} \gg c_{A 0}$ [see Eq. (9)]. (right) Representation in logarithmic units when $c_{A 0}=c_{B 0} / 2=1.5 \times 10^{-4} \mathrm{~mol}^{-1}$ [stoichiometric case, see Eq. (8) with $t_{c}=300 \mathrm{~s}$ ]. (c) Kinetic curves for different initial B concentrations. (d) Inverse proportionality of $t_{c}$ to $c_{B 0}$. (e) Dependence of $t_{c}$ on the solution $\mathrm{pH}\left(T=23^{\circ} \mathrm{C}\right)$. (f) Dependence of $t_{c}$ on the solution temperature $(\mathrm{pH}=12)$. (g) Summary of kinetics dependencies in water. (h) Dependence of $t_{c}$ on the solution viscosity $\eta$. 
The reaction rate is sensitive to the medium $\mathrm{pH}$ and is thermally activated, and we find, in water [Figs. 2(a)-2(g)]

$$
k=k_{0} \exp \left(\mathrm{pH}-\frac{E}{R T}\right),
$$

where $k_{0}=1.67 \mathrm{~mol}^{-1} 1 \mathrm{~s}^{-1}, E=24 \mathrm{~kJ} \mathrm{~mol}^{-1}$ is the activation energy, and $R=8.31 \mathrm{~J} \mathrm{~mol}^{-1} \mathrm{~K}^{-1}$ is the gas constant. The reaction slows down in a more viscous substrate [Fig. 2(h)].

Reasonable production times are obtained by using a large excess of $\mathrm{B}$ on $\mathrm{A}$, namely, $c_{B 0} \gg c_{A 0}$. In that case, the reaction kinetics is of a pseudo-first-order type so that

$$
\dot{c}=\frac{c_{A}}{t_{c}},
$$

with $t_{c}$ given in (10) and we have $c=c_{A 0}\left(1-e^{-t / t_{c}}\right)$, as seen from (9) above, and in Figs. 2(a) and 2(b).

\section{EXPERIMENTS}

To benchmark and demonstrate the capabilities of this reaction for mixing studies, we present two reaction-diffusion experiments. The two experiments are both in a Hele-Shaw cell. In the first, the reaction front propagates in the cell plane, while in the second, it develops across the fluid layer transverse to the cell plane. These two configurations complement each other and are equally necessary for qualifying the reaction properly. In both cases, the mixing time $t_{s}$ is a diffusion time, constructed on the appropriate length scale. In what follows, the working fluid (a mixture of water and UCON oil [34]) has a viscosity $7 \times 10^{-3} \mathrm{~Pa} \mathrm{~s}$, setting the species diffusion coefficients $D_{i}$ listed in Table II. The solutions $\mathrm{pH}=12$, temperature $T=23^{\circ} \mathrm{C}$, and $c_{A 0}=3 \times 10^{-5} \mathrm{moll}^{-1}$ are kept constant. Density and viscosity contrasts between the phases are negligible [35].

\section{A. Planar reaction-diffusion fronts}

The first experiment aims at contacting A with B along a straight front with initial controlled thickness. We use a Hele-Shaw cell made of two 200-mm-square Plexiglas plates spaced by a 0.2mm-thick sheet of latex cut in a diamond-shaped mask [Fig. 3(a)]. The cell is fitted with two inlets and two outlets at the corners of the diamond; the inlets, facing each other in the $x$ direction for the injection of the reactants, and the two outlets for the symmetrical evacuation of the flow in the $y$ direction. The engineering of this setup has been introduced in Ref. [36]. The solutions of the reactants $\mathrm{A}$ and $\mathrm{B}$ are pushed at the same flow rate in the inlets by a syringe pump. They meet at the center of the cell where a divergence-free $(\nabla \cdot \mathbf{u}=0)$ stagnation point flow is formed with velocity field $\mathbf{u}=(-\gamma x, \gamma y)$ whose compression rate $\gamma$ (of order $10^{-2} \mathrm{~s}^{-1}$ ) depends on the geometry of the cell, and on the intensity of the imposed inlet flow rate. There, a steady compressed (in $x$ ) stretched (in $y$ ) front is formed separating the reactants which interpenetrate, react and release $\mathrm{F}$ which in turn diffuses away from the front as a uniform (along $y$ because $\partial c / \partial y \ll \partial c / \partial x$ ) thin strip whose width (along $x$ ) is given by the Batchelor [37] scale $\ell$ which balances the rate of diffusive spreading $D_{F} / \ell^{2}$ with the substrate compression rate $\gamma$ as $\ell \sim \sqrt{D_{F} / \gamma}$. There is a different $\ell$ for each species when the diffusion coefficients $D_{i}$ are different (see Table II), but since these are all of the same order of magnitude, the one constructed with $D_{F}$ is representative for all species. That thickness $\ell$ (of order $0.1 \mathrm{~mm}$ ) which is a measure of the steepness of the species concentration gradient at the front also sets the initial value of Da since, with $t_{s}=\ell^{2} / D_{A}$ in that case (see Ref. [4]), we have from (1)

$$
\mathrm{Da} \approx \frac{1}{\gamma t_{c}}
$$

where we have made $D_{A} \approx D_{F}$. Once $\ell$ (and therefore Da) has been set to the desired value, the injection is stopped and the drain holes are plugged to prevent leakage; the medium is immobile. 


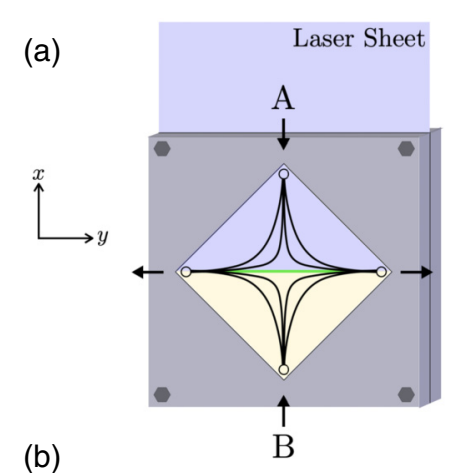

(b)

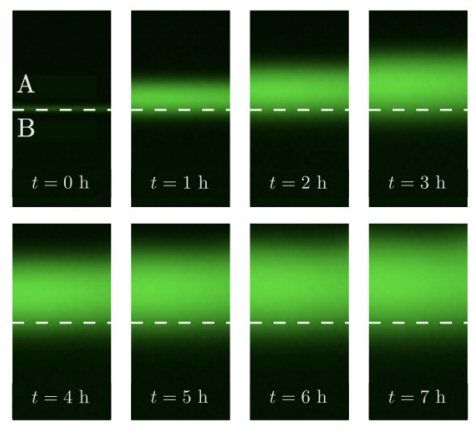

(c)
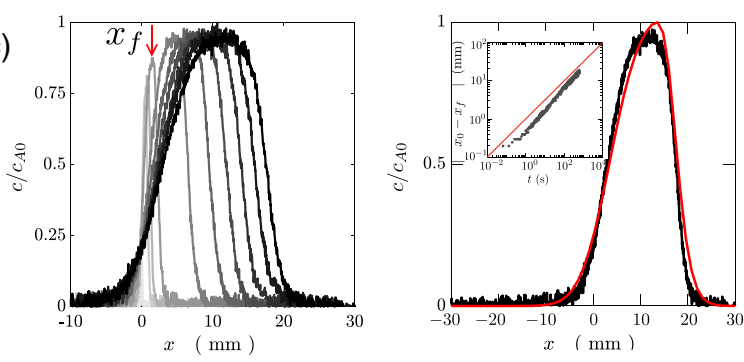

(d)
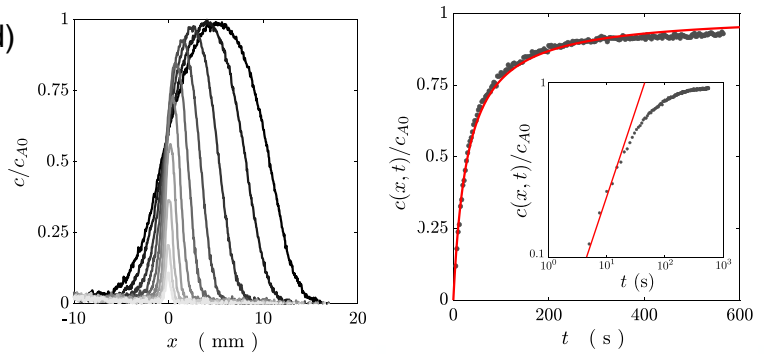

(e)

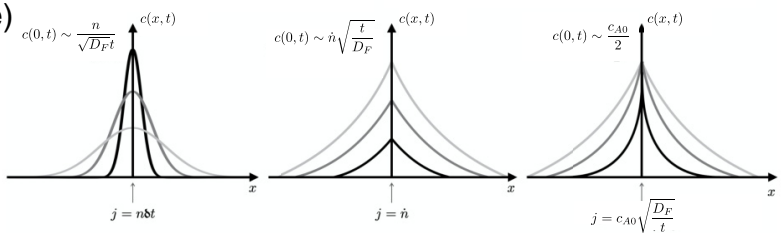

FIG. 3. Planar reaction-diffusion front. (a) Schematic of the experimental setup featuring a stagnation line where reactants $\mathrm{A}$ and $\mathrm{B}$ meet. (b) Development of the product $\mathrm{F}$ front at different times after contact; $c_{A 0}=$ $3 \times 10^{-5} \mathrm{moll}^{-1}, c_{B 0}=3 \times 10^{-1} \mathrm{~mol}^{-1}$. (b) Fast reaction: concentration profiles of $\mathrm{F}$ at $t=1,2,4,8,16,32$, 64, 128, 256, and $512 \mathrm{~min}$ (left) and (right) comparison with (26) (red line). The inset shows the location $x_{f}(t)$ of the production source (18). (d) Slow reaction: concentration profiles of $\mathrm{F}$ at $t=1,2,4,8,16,32,64,128$, 256, and 512 minutes (left). Measured peak product concentration compared with $c\left(x_{f}, t\right)=c_{A 0}\left(1-e^{-t / t_{c}}\right)$ (red line). The inset shows the logarithmic scale emphasizing that $c\left(x_{f}, t\right) \sim c_{A 0} t / t_{c}$ at short times (right). (e) Schematic representations of the concentration product diffusion front for various reactant fluxes $j$.

Then, the evolution of the $\mathrm{F}$ concentration profile is recorded thanks to a $488 \mathrm{~nm}$ laser illumination sheet slicing the cell along the cell gap [Fig. 3(b)]. The profiles shown in Figs. 3(c) and 3(d) are averages of the $\mathrm{F}$ concentration profiles performed along the $y$ direction over a distance of about $30 \mathrm{~mm}$ around the center of the cell where the front is perfectly flat.

\section{Fast reaction}

We first consider the fast version of the reaction with $\mathrm{Da}=12$ obtained for $c_{B 0}=3 \times$ $10^{-1} \mathrm{moll}^{-1}$ [see Fig. 3(c)]. After a short transient, the profile $c(x, t)$ peaks at a constant value whose location $x_{f}(t)$ increases at the same rate as the whole profile broadens. This is typical of the extensively studied large-Da limit [24,25,27-29,38-40] where a tiny reaction zone is a pure sink for the reactants, reaching it by diffusion, which therefore limits the chemical production rate: The thickness $\xi_{\star}$ of the reaction zone, and the typical reactant concentration in it, $c_{\star}$, scale as

$$
\xi_{\star} \sim \xi \mathrm{Da}^{-1 / 3} \text { and } c_{\star} \sim c_{A 0} \mathrm{Da}^{-1 / 3},
$$

both vanishing as $\mathrm{Da}=\left(\xi^{2} / D_{A}\right) / t_{c} \rightarrow \infty$ where $\xi$ is a so-called outer scale $[25,41]$ given by the diffusional thickness of the reactants profile. For instance, with $\xi \sim \sqrt{D_{A} t}$, one recovers the $\xi_{\star} \sim$ $t^{1 / 6}$ growth of the reaction zone thickness derived in Ref. [25]. 

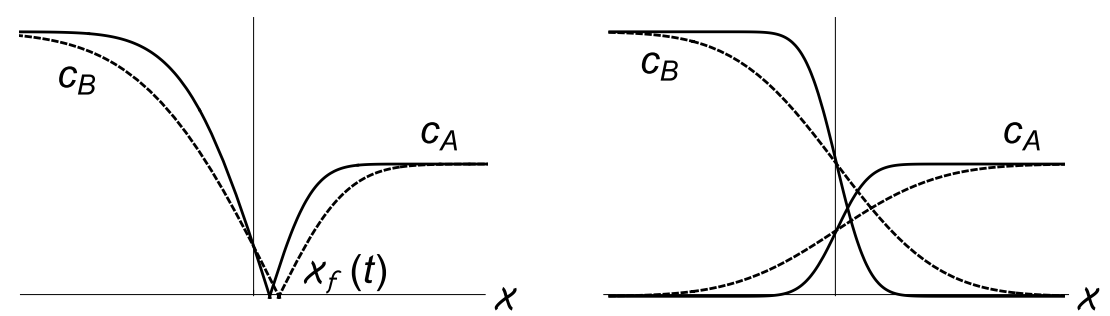

FIG. 4. Sketches of the reactants A and B concentration profiles shown at two instants of time. (left) In the limit of fast chemistry, defining the reactive interface location $x_{f}(t)$. (right) In the slow-chemistry limit, illustrating the diffusive interpenetration of the phases prior to reaction. In the latter case, the initial net production rate $k \int c_{A} c_{B} d x=k c_{A 0} c_{B 0} \sqrt{2 D t / \pi} \sim\left(c_{A 0} / t_{C}\right) \xi(t)$ is extensive to the diffusive length $\xi(t) \sim \sqrt{D t}$.

In the $\mathrm{Da} \rightarrow \infty$ limit, the reactants sink is, conversely, a localized source for the product F. From this fact, we proceed with a number of predictions concerning the location of the source, and the resulting product concentration profile.

The diffusion flux of B towards the sink is of order $D_{B} c_{B 0} / \sqrt{D_{B} t}$ while that of $\mathrm{A}$ is $D_{A} c_{A 0} / \sqrt{D_{A} t}$. Since, owing to the reaction stoichiometry one mole of A reacts with one of B per unit $t_{c}$, the location $x_{f}(t)$ of the sink (Fig. 4) must move to compensate for the diffusion flux contrast according to

$$
\begin{gathered}
D_{B} \frac{c_{B 0}}{\sqrt{D_{B} t}}-c_{B 0} \dot{x_{f}}(t)=D_{A} \frac{c_{A 0}}{\sqrt{D_{A} t}}+c_{A 0} \dot{x_{f}}(t), \text { or } \\
x_{f}(t)=\alpha \sqrt{D_{B} t}, \text { with } \alpha=\frac{c_{B 0}-c_{A 0} \sqrt{D_{A} / D_{B}}}{c_{A 0}+c_{B 0}} .
\end{gathered}
$$

The condition for front stationarity $(\alpha=0)$ is far from being met here since $c_{B 0} \gg c_{A 0}[27,42]$.

To understand the shape of the product concentration profile $c(x, t)$, let us first consider the simplest version when the front is stationary, fed in $x=0$ by a (symmetrical) reactants flux $j(t)$. On a still substrate, we have

$$
\partial_{t} c=D_{F} \partial_{x}^{2} c+j(t) \delta(x)
$$

where $\delta(x)$ denotes the Dirac delta function. The net amount of $\mathrm{F}$ formed at time $t$ per unit front area is

$$
n_{F}=\int_{-\infty}^{\infty} c(x, t) d x
$$

and from (19) we have $\dot{n_{F}}(t)=j(t)$. Let us examine some simple standard forms for $j(t)$ [see, e.g., Ref. [43] for detailed solutions, and Fig. 3(e)].

(1) If $n$ molecules of $\mathrm{F}$ are released impulsively at $t=0$, then $j(t)=n \delta(t)$ and

$$
c(x, t) \sim \frac{n}{\sqrt{D_{F} t}} e^{-\frac{x^{2}}{4 D_{F} t}},
$$

giving $c(0, t) \sim n / \sqrt{D_{F} t}$ and $n_{F}(t)=n$.

(2) If the molecule flux is constant, then $j(t)=\dot{n}$ is independent of time, thus $c(0, t) \sim \dot{n} \sqrt{t / D_{F}}$ and $n_{F}(t)=\dot{n} t$.

(3) If the flux is decaying in time like, e.g., $j(t)=c_{A 0} \sqrt{D_{F} / t}$, then $c(0, t)=c_{A 0} / 2$ and $n_{F}(t)=$ $c_{A 0} \sqrt{D_{F} t}$.

Since the product can leave the source location by diffusion only, its exploration distance is typically $\sqrt{D_{F} t}$ at time $t$, and the maximal concentration $c(0, t)$ adjusts to comply to mass 
conservation. We thus have in general

$$
n_{F}(t)=\int_{0}^{t} j\left(t^{\prime}\right) d t^{\prime} \sim c(0, t) \sqrt{D_{F} t}
$$

from where the three particular cases and the scaling laws for $c(0, t)$ and $n_{F}(t)$ discussed above derive.

In the present problem, similar to case 3 listed above, the product $\mathrm{F}$ is deposited at a decreasing rate $j\left(x_{f}\left(t^{\prime}\right), t^{\prime}\right) \sim c_{A 0} \sqrt{D_{A} / t^{\prime}}$ in $x=x_{f}\left(t^{\prime}\right)$ given in Eq. (18) above, now varying in time, and diffuses away from the location where it has been produced. The overall concentration profile is thus a superposition of all the contributions from temporally varying production locations, and flux. We may formalize this superposition operation using the Green's function [43] of the diffusion equation $\mathcal{G}\left(x, t, x^{\prime}, t^{\prime}\right)$ which represents the concentration profile of a unitary quantity deposited impulsively in $x^{\prime}$ at time $t^{\prime}$ as

$$
\begin{gathered}
c(x, t)=\int_{-\infty}^{\infty} d x^{\prime} \int_{0}^{t} d t^{\prime} \mathcal{G}\left(x, t, x^{\prime}, t^{\prime}\right) j\left(x^{\prime}, t^{\prime}\right), \\
\mathcal{G}\left(x, t, x^{\prime}, t^{\prime}\right)=\frac{1}{2 \sqrt{\pi D_{F}\left(t-t^{\prime}\right)}} e^{-\frac{\left(x-x^{\prime}\right)^{2}}{4 D_{F}\left(t-t^{\prime}\right)}}, \\
j\left(x^{\prime}, t^{\prime}\right) \approx c_{A 0} \sqrt{\frac{D_{F}}{t^{\prime}}} \delta\left(x^{\prime}-x_{f}\left(t^{\prime}\right)\right),
\end{gathered}
$$

leading to

$$
c(x, t) \approx c_{A 0} \int_{0}^{t} d t^{\prime} \frac{1}{2 \sqrt{\pi t^{\prime}\left(t-t^{\prime}\right)}} e^{-\frac{\left[x-x_{f}\left(t^{\prime}\right)\right]^{2}}{4 D_{F}\left(t-t^{\prime}\right)}},
$$

which is integrated numerically with $x_{f}(t)$ given in (18). The maximal F concentration $c\left(x_{f}, t\right) \approx$ $c_{A 0}$ is constant in time because the product diffuses away from the location where it is formed at the same rate $\left(\sim c_{A 0} \sqrt{D_{F} / t}\right)$ it is produced by the incoming limiting reactant $\mathrm{A}$, as seen in Fig. 3(c), which also shows that the overall product profile is well represented by (26).

\section{Slow reaction}

More subtle is the slow-reaction version of the problem, with $\mathrm{Da}=0.04 \ll 1$ obtained for $c_{B 0}=$ $3 \times 10^{-4} \mathrm{moll}^{-1}$ [see Fig. 3(d)]. In that case the transient from $c=0$ to $c=c_{A 0}$ is itself slow, featuring an increase precisely described by the pure chemical kinetics limit, within an overall front broadening and shifting diffusively, as before. The concentration [see (7)] peaks at

$$
c\left(x_{f}, t\right)=c_{A 0}\left(1-e^{-t / t_{c}}\right) .
$$

Diffusion broadening is faster than chemical consumption when $\mathrm{Da}<1$, meaning that the species interpenetrate diffusively before they have appreciably reacted. The (initially purely diffusive, see Fig. 4) reactants fields is $c_{i}(x, t) / c_{i 0}=\frac{1}{2}\left[1+\operatorname{erf}\left( \pm x / 2 \sqrt{D_{i} t}\right)\right]$ so that the net production rate $k \int c_{A} c_{B} d x=k c_{A 0} c_{B 0} \sqrt{2 D t / \pi} \sim\left(c_{A 0} / t_{c}\right) \xi(t)$ is of order $c_{A 0} / t_{c}$ as if the reactants were initially premixed [see, e.g., (14) and (27)], and is extensive to the diffusive interpenetration length $\xi(t) \sim \sqrt{D t}$ with $D=D_{A}+D_{B}$.

Within $\delta t^{\prime}$, the reaction volume (per front area) has thus increased by an amount $\delta \xi\left(t^{\prime}\right) \sim$ $\sqrt{D / t^{\prime}} \delta t^{\prime}$ and in this volume, the reaction has produced $\delta n_{F}\left(t-t^{\prime}\right)=c\left(x_{f}, t-t^{\prime}\right) \delta \xi\left(t^{\prime}\right)$ moles of $\mathrm{F}$ during the time interval $t-t^{\prime}$. The net amount of $\mathrm{F}$ formed at time $t$ is thus

$$
\begin{aligned}
n_{F}(t) & \approx c_{A 0} \int_{0}^{t}\left(1-e^{-\frac{t-t^{\prime}}{t_{c}}}\right) \sqrt{\frac{D}{t^{\prime}}} d t^{\prime} \\
& \sim c_{A 0} \frac{t}{t_{c}} \sqrt{D t} \text { for } \frac{t}{t_{c}}<1,
\end{aligned}
$$




$$
\text { and } \sim c_{A 0} \sqrt{D t} \text { for } \frac{t}{t_{c}}>1
$$

We call diffusio-chemical the early-time regime when $t / t_{c}<1$ because the production

$$
c_{A 0}\left(1-e^{-t / t_{c}}\right) \stackrel{t / t_{c} \ll 1}{\longrightarrow} c_{A 0} \frac{t}{t_{c}}
$$

is limited by chemical kinetics in a volume growing proportionally to $\sqrt{D t}$ which is itself the result of a diffusive interpenetration. The product concentration $c=n_{F}(t) / \sqrt{D t}$ in the interpenetration volume scales as $c_{A 0} t / t_{c}$. The large time regime $t / t_{c}>1$ amounts to the fast reaction regime above, since, in these late times, the diffusion flux has decayed enough to limit the production rate of $\mathrm{F}$, whose concentration is $n_{F}(t) / \sqrt{D t}=c_{A 0}$. The crossover occurs for $t=t_{c}$ or (with $\xi=\sqrt{D t}>\ell$, see Fig. 3)

$$
\mathrm{Da}=\frac{\xi^{2} / D}{t_{c}}=1 .
$$

Because the diffusion flux from a step profile between segregated reactants diverges (like $1 / \sqrt{t}$ ) at short time, their bimolecular, finite kinetics chemical transformation necessarily limits the production rate at early time.

\section{B. Reaction from a confined diffusive lens}

The inspiration of the second experiment is similar to the first, but now in a confined geometry since we study the development of the reactive front across the gap of the cell. We use a circular Hele-Shaw cell made by two circular 200-mm-diameter Plexiglass ${ }^{\circledR}$ plates spaced by $H=0.75 \mathrm{~mm}$. The gap is entirely filled with $\mathrm{B}$. The center of the upper plate is drilled by a $2 \mathrm{~mm}$ hole for the injection of $\mathrm{A}+\mathrm{Rh}$. The passive rhodamine $\left(c_{R h}=1 \times 10^{-6} \mathrm{moll}^{-1}\right)$ does not interfere with the chemical reaction between $\mathrm{A}$ and $\mathrm{B}$ and serves as a tracer to check the kinematics of the flow. The cell is illuminated in volume using an argon-ion laser emitting at $488 \mathrm{~nm}$ (exciting the fluorescence of F) and $514 \mathrm{~nm}$ (exciting Rh). The $\mathrm{A}+\mathrm{Rh}$ solution is injected at a flow rate $q$ for a time $t_{i n j}$ (in the range 10-1000 s) to form a wide lens with radius $R \approx 40 \mathrm{~mm}$ surrounded by the invaded $\mathrm{B}$. We vary $c_{B 0}$ to vary the reaction time $t_{c}$.

The radial (along $r$ ) thickness profile of the $\mathrm{A}+\mathrm{Rh}$ lens across the gap is, as shown from the passive Rh fluorescence field [Fig. 5(c)], parabolic, of a Poiseuille-type, somewhat smeared by Taylor dispersion [44] during the injection phase; this is particularly apparent close to the injection point where the B layer is thin, and near the lens extremity where diffusion develops along two degrees of freedom (radial and transverse). The kinematic radial velocity $u(r, x)$ at elevation $x$ is

$$
u(r, x) \sim \frac{q}{r H}\left\{1-[x /(H / 2)]^{2}\right\}
$$

so that the radial extent of the lens such that $\dot{R}=u(R, 0)$ is $R(t) \sim \sqrt{q t / H}[18,45]$. Given the volume $\Omega=q t_{i n j}$ injected, its maximal radial extent $R^{2} \sim \Omega / H$ is such that the lens aspect ratio is $H / R \ll 1$. Therefore, the interface between the two phases is, for $r \ll R$, practically aligned with the vertical direction $x$, along which the reaction-diffusion front develops in a quasi one-dimensional fashion $\left(\partial C_{i} / \partial r \ll \partial C_{i} / \partial x\right)$. The fluorescence signal recorded perpendicularly to the cell plane gives access to the net quantity of $\mathrm{F}$ produced at a given radius $r$, integrated over the gap thickness

$$
n_{F}(r, t)=\int_{-H / 2}^{H / 2} c(r, x, t) d x .
$$

After injection, fluorescence increases everywhere on the invaded area $\pi R^{2}$, except within a region of radius $r_{\star}$. Close to the injection point as $r \rightarrow 0$, the thickness $h(r, t)$ of the close-toimmobile B phase at the cell wall is vanishingly small, given by (35). The thin B layer there has 


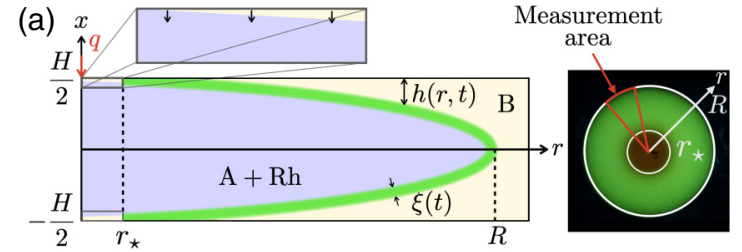

(b)
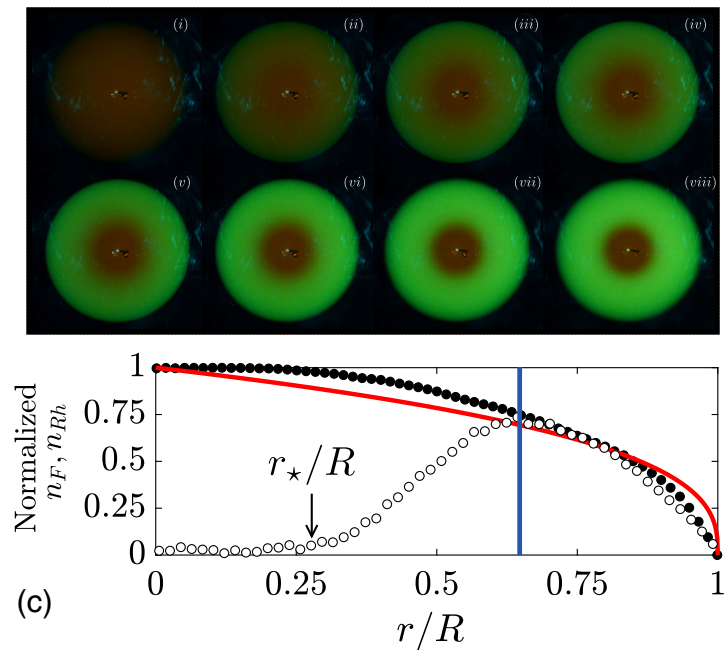

(d)

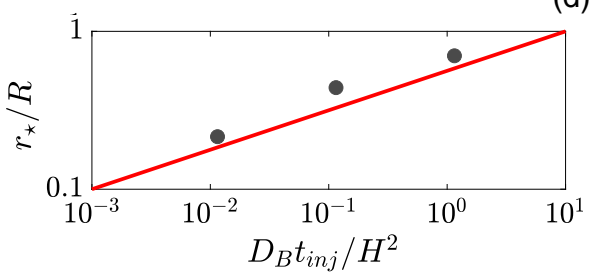

(e)

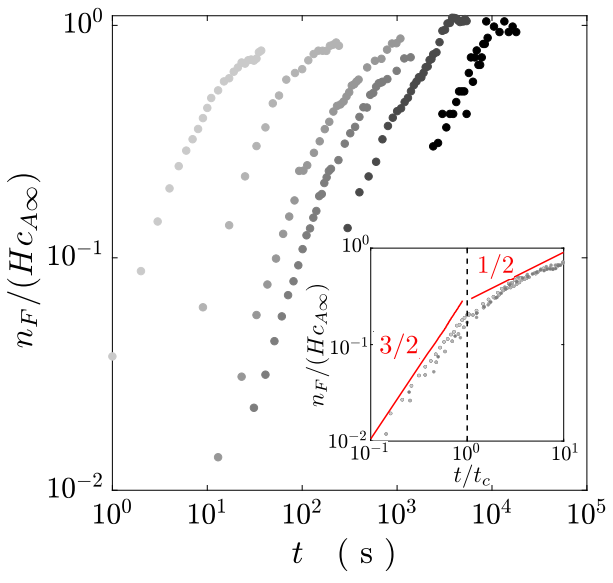

FIG. 5. Reaction from a confined diffusive lens. (a) Schematic of the experimental setup seen from the side (left) and from the top (right). (b) Gap integrated concentration patterns of $\mathrm{F}$ for, successively, $t=0$, $1,2,3,5,10,20$, and $30 \mathrm{~min} ; c_{A 0}=3 \times 10^{-5} \mathrm{moll}^{-1}, c_{B 0}=3 \times 10^{-3} \mathrm{~mol}^{-1}$. (c) Radial gap integrated concentration profiles of Rh (black dots, and red line fit by a kinematic Poiseuille displacement field) and F (white dots). The blue line represents the radius at which the measurements are performed. (d) "Washing" radius $r_{\star}$ as a function of the injection time $t_{i n j}$ and prediction from (35). (e) Net product $n_{F}(r / R \approx 0.6, t)$ as a function of time for $c_{B 0}=3 \times 10^{-2}, 1.5 \times 10^{-2}, 3 \times 10^{-3}, 1.5 \times 10^{-3}, 3 \times 10^{-4}$, and $3 \times 10^{-5} \mathrm{~mol}^{-1}$; $c_{A 0}=3 \times 10^{-5} \mathrm{moll}^{-1}$. The inset shows the integrated net product $n_{F}$ as a function of $t / t_{c}$, highlighting the transition between the diffusio-chemical and the diffusive regime at $t / t_{c}=1 ; c_{B 0}=3 \times 10^{-2}, 1.5 \times 10^{-2}$, $3 \times 10^{-3}, 1.5 \times 10^{-3} \mathrm{moll}^{-1}$.

thus time to diffuse towards the more rapid layers at the symmetrical plane of the cell, via the Taylor mechanism [44], during the injection phase. That layer is therefore "washed," incorporated in the liquid bulk. No reaction can thus occur at the corresponding $r$ location as long as $h(r, t)^{2} / D_{B}<t_{\text {inj }}$, a condition defining a region with radius $r_{\star}$ such that

$$
h(r, t) \approx \frac{\pi}{6} \frac{H^{2} r^{2}}{q t}, \text { giving } \frac{r_{\star}}{R} \sim\left(\frac{D_{B} t_{i n j}}{H^{2}}\right)^{1 / 4} .
$$

At larger radial locations $r>r_{\star}$, where the B layer is thick enough to allow for the species to react as they face each other, the net product $n_{F}(r, t)$ increases in time according to two distinct regimes, first a rapid $t^{3 / 2}$ increase typical of the diffusio-chemical regime, followed by the purely diffusive $t^{1 / 2}$ regime, both analyzed in (29) and (30). The crossover occurs, as expected, at $t / t_{c}=1$, as seen from Fig. 5(e) featuring experiments with a broad range of $t_{c}$. The diffusive regime has already been studied in this radial geometry, using a different chemical reaction [18].

The confined geometry with a finite gap thickness $H$ allows us to explore an additional regime, namely, the pure chemical regime. This regime onsets for very large $t_{c}$ (slow chemistry) at times larger than $H^{2} / D$, when the diffusive interpenetration distance $\xi(t)$, and reaction zone thickness $\xi_{\star}$ 

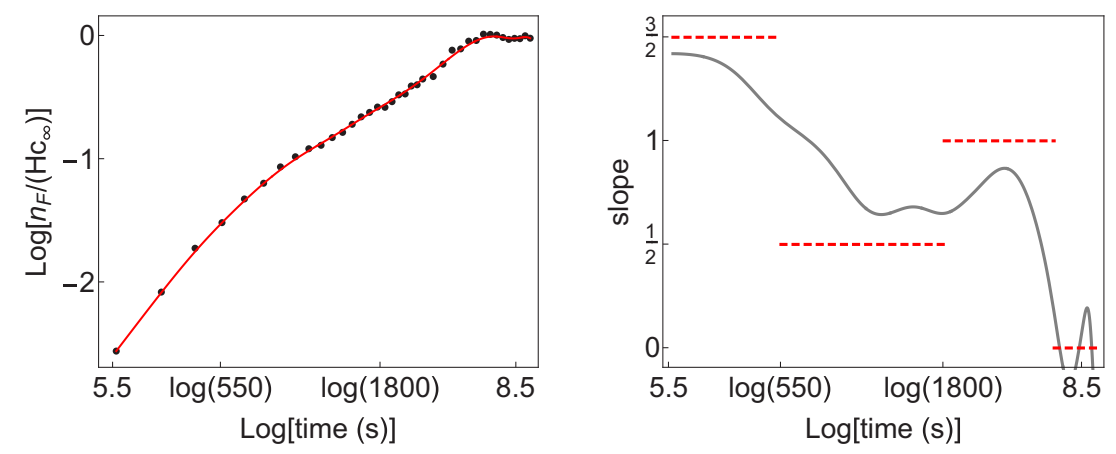

FIG. 6. (left) Net product $n_{F}(r / R \approx 0.5, t)$ as a function of time measured in a single experiment spanning the three successive regimes (diffusio-chemical, diffusive, chemical) before the exhaustion of reactant $\mathrm{A} ; c_{B 0}=$ $3 \times 10^{-4} \mathrm{~mol}^{-1}$. (right) Local logarithmic slope $d \ln \left(n_{F}\right) / d \ln (t)$ as a function of time, showing an oscillation within the expected bounds $3 / 2$ (diffusio-chemical), $1 / 2$ (diffusive), 1 (chemical), and 0 (reaction completed) in each sub-regime, and the associated crossover times $t_{c} \approx 550 \mathrm{~s}$ and $H^{2} / D \approx 1800 \mathrm{~s}$.

become comparable to $H$. In this ultimate stage, chemistry fully controls the production kinetics with concentrations of $\mathrm{A}$ and $\mathrm{B}$ resulting from the dilution of the species across the cell gap as $c_{A \infty}=c_{A 0}\left[1-h\left(r, t_{i n j}\right)\right] / H$ and $c_{B \infty}=c_{B 0} h\left(r, t_{i n j}\right) / H$ and where we expect, owing to Eq. (31) that

$$
n_{F}(t) \sim c_{A \infty} \frac{t}{t_{c}} H
$$

at the onset of this regime, with $t_{c}$ computed from $c_{B \infty}$. Figure 6 shows how the three consecutive regimes could be recovered from a single experiment lasting for more than an hour and a half with $t_{c} \approx 550 \mathrm{~s}$ and $H^{2} / D \approx 1800 \mathrm{~s}$. While the distinct power laws are not crystal clear in such narrow time intervals, the trends are, and are not incompatible with the anticipated scalings in each subregime. The crossovers occur when expected (namely, about $t_{c}$ and $H^{2} / D$ ).

\section{SUMMARY: KINETIC REGIMES}

We have introduced and documented an original chemical reaction producing an easily diagnosable, fluorescent product in water (fluorescein). The reaction has a broadly tunable chemical kinetics allowing the quantitative investigation of the interplay between molecular diffusion and reaction kinetics in various limits. Specifically, for a bimolecular pseudo-first-order reaction with chemical time $t_{c}$ between initially segregated reactants with mutual diffusion coefficient $D$ separated by a sharp flat front in an enclosure of size $H$, we have shown that the amount of product per unit front area and initial reactant concentration $c_{0}$ is

$$
\begin{aligned}
n_{F}(t) / c_{0} & \sim \frac{t}{t_{c}} \sqrt{D t} \text { for } t<t_{c} \\
& \sim \sqrt{D t} \text { for } t>t_{c} \\
& \sim \frac{t}{t_{c}} H \text { for } t>t_{c}>H^{2} / D .
\end{aligned}
$$

To each transition is associated an order-unity Damköhler number $\mathrm{Da}=\left(\xi^{2} / D\right) / t_{c}$, with $\xi \sim \sqrt{D t}$ for the transition $(i)-(i i)$, and $\xi=H$ for the (ii)-(iii) transition. In particular, besides the known chemical (iii) and diffusion-limited (ii) regimes, we have identified the early time diffusio-chemical regime $(i)$ where the production rate is limited by chemical kinetics, in a volume increasing by diffusion when the diffusive broadening rate overcomes the one of chemical conversion. 

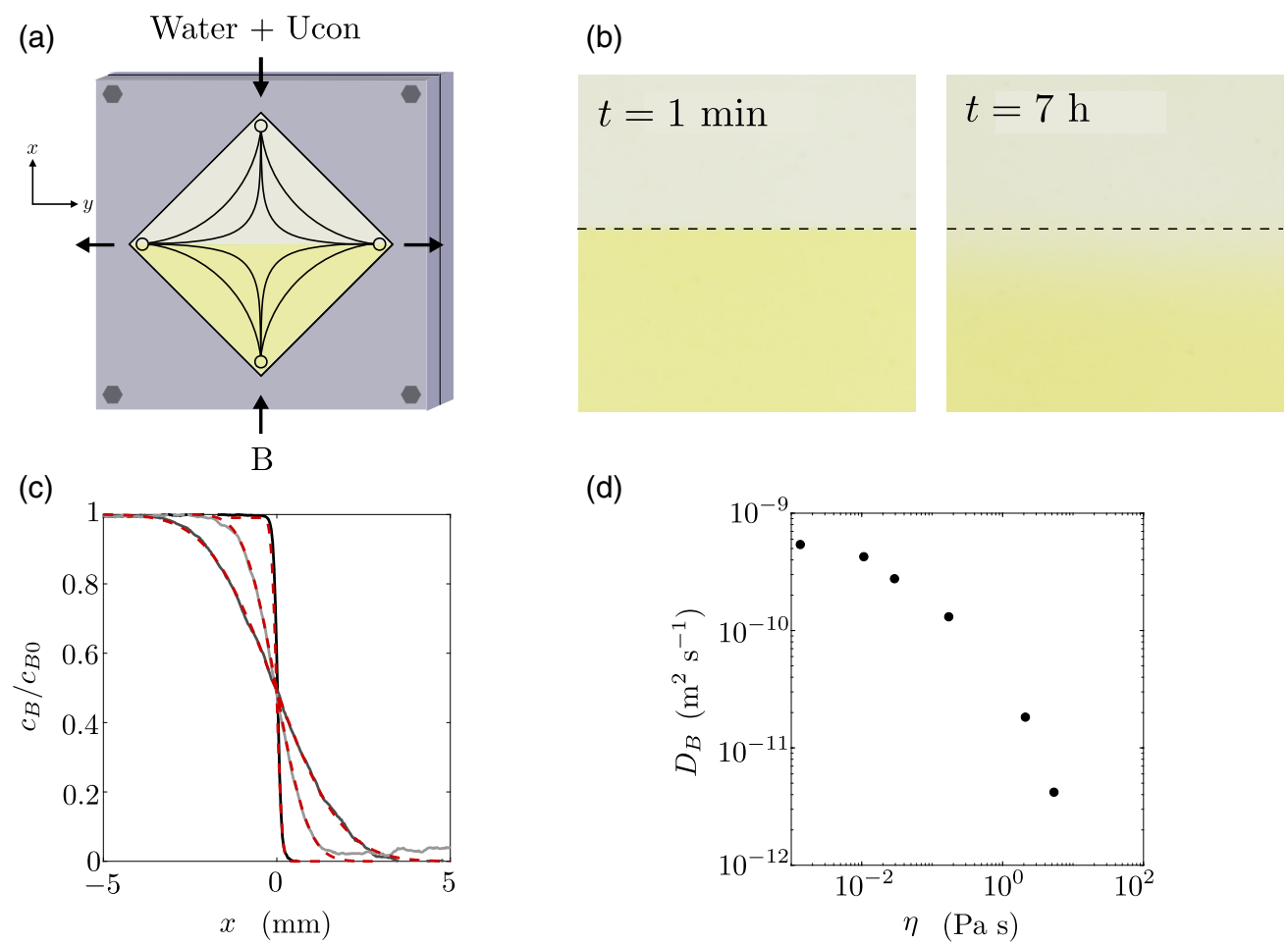

FIG. 7. (a) Schematic showing the stagnation-point method to form an initial sharp front between a solution of B and a clear solution (mixtures of water and UCON oil). (b) Development of the diffusive interpenetration between a solution of $\mathrm{B}$ and a mixture of water and UCON at rest, for two successive times $\left(c_{B 0}=6 \times 10^{-2} \mathrm{moll}^{-1}, \eta=0.2 \mathrm{~Pa} \mathrm{~s}\right)$. (c) Experimental profiles $c_{B}(x, t)$ at $t=1,225$, and $500 \mathrm{~min}$ (solid lines) and fit by Eq. (A1) (dashed lines). (d) Diffusion coefficient of potassium ferricyanide $D_{B}$ as a function of the solution viscosity.

These studies, which were conducted in Hele-Shaw cells with still substrates are currently extended to situations where the substrate is stirred [46]. The chemical reaction documented here will, for instance, tell where and for how long the reactants have interpenetrated at the diffusive scale, what is the impact of stirring on the product mixture's composition [47] in any stirred, even partially mixed mixture.

\section{APPENDIX: THE DIFFUSION COEFFICIENT $D_{B}$ OF POTASSIUM FERRICYANIDE IN AQUEOUS SOLUTIONS}

The diffusion coefficient of potassium ferricyanide (B) was measured in the same experimental setup [shown in Fig. 7(a)] as the one presented in Sec. III: it consists in recording the broadening of a one-dimensional planar diffusion front of $\mathrm{B}$ in aqueous solutions at rest as time proceeds. The densest solution of $\mathrm{B}$ is placed below the clear solution of the same liquid (mixtures of UCON oil and water) in a back-lighted vertical Hele-Shaw cell. As the two phases interpenetrate diffusively, the vertical transmitted intensity profiles $I(x, t)$ are recorded at successive instants of time. Potassium ferricyanide has a yellowish color in aqueous solution, and absorbs white light. The local intensity $I(x, t)$ transmitted through the cell reflects the local concentration $c_{B}(x, t)$ through 
the Beer-Lambert law

$$
c_{B}(x, t)=-\frac{1}{\epsilon l} \ln \left(\frac{I(x, t)}{I_{0}}\right),
$$

with $\epsilon$ the molar absorptivity and $l$ the optical path length (the cell thickness). Figure 7(b) shows that the flat concentration front is initially sharp, when after seven hours it presents the blurry aspect characteristic of diffusive broadening. Adjusting the anticipated diffusive response

$$
\frac{c_{B}(x, t)}{c_{B 0}}=\frac{1}{2} \operatorname{erfc}\left(\frac{x}{2 \sqrt{D_{B} t}}\right),
$$

which is indeed found to be appropriate [Fig. 7(c)], to the measured profiles with $D_{B}$ as the only fitting parameter constitutes a measurement of $D_{B}$ itself. Experiments performed with solution of different UCON volume fractions allow the diffusion coefficient of potassium ferricyanide to be known empirically for viscosities in aqueous solutions ranging from $\eta=10^{-3} \mathrm{~Pa}$ s to $\eta=10^{2} \mathrm{~Pa} \mathrm{~s}$ [see Fig. 7(d)].

[1] D. A. Frank-Kamenetskii, Diffusion and Heat Transfer in Chemical Kinetics (Plenum Press, New York, 1969).

[2] M. Boudart, Kinetics of Chemical Processes (Prentice-Hall, Inc., 1968).

[3] G. Damköhler, Der Einfluss der Turbulenz auf die Flammengeschwindigkeit in Gasgemischen, Z. Elektrochem. Angew. Phys. Chem. 46, 601 (1940).

[4] E. Villermaux, Mixing versus stirring, Annu. Rev. Fluid Mech. 51, 245 (2019).

[5] P. V. Danckwerts and R. A. M. Wilson, Flow-visualization by means of a time-reaction, J. Fluid Mech. 16, 412 (1963).

[6] J. R. Bourne, C. Hilber, and G. Tovstiga, Kinetics of the azo coupling reactions between 1-naphthol and diazotised sulphanilic acid, Chem. Eng. Commun. 37, 293 (1985).

[7] R. J. Demyanovich and J. R. Bourne, Rapid micromixing by the impingement of thin liquid sheets. 2. Mixing study, Ind. Eng. Chem. Res. 28, 830 (1989).

[8] R. Breidenthal, Structure in turbulent mixing layers and wakes using a chemical reaction, J. Fluid Mech. 109, 1 (1981).

[9] A. R. Karagozian, Y. Suganuma, and B. D. Strom, Experimental studies in vortex pair motion coincident with a liquid reaction, Phys. Fluids 31, 1862 (1988).

[10] B. M. Cetegen and N. Mohamad, Experiments on liquid mixing and reaction in a vortex, J. Fluid Mech. 249, 391 (1993).

[11] W. J. A. Dahm and P. E. Dimotakis, Measurements of entrainment and mixing in turbulent jets, AIAA J. 25, 1216 (1987).

[12] A. F. Corriveau and W. D. Baines, Difusive mixing in turbulent jets as revealed by a pH indicator, Exp. Fluids 16, 129 (1993).

[13] A. Douhal, F. Lahmani, and A. H. Zewail, Proton-transfer reaction dynamics, Chem. Phys. 207, 477 (1996).

[14] O. F. Mahammed, D. Pines, E. Pines, and E. T. J. Nibbering, Aqueous bimolecular proton transfer in acid-base neutralization, Chem. Phys. 341, 240 (2007).

[15] E. Villermaux, Fast bimolecular reactions in high Reynolds number turbulence: Structure of the reactive interface and surface of reaction, in Advances in Turbulence V (Springer, 1995), pp. 529-533.

[16] R. F. Ismagilov, A. D. Stroock, P. J. A. Kenis, G. Whitesides, and H. A. Stone, Experimental and theoretical scaling laws for transverse diffusive broadening in two-phase laminar flows in microchannels, Appl. Phys. Lett. 76, 2376 (2000).

[17] C. N. Baroud, F. Okkels, L. Ménétrier, and P. Tabeling, Reaction-diffusion dynamics: Confrontation between theory and experiment in a microfluidic reactor, Phys. Rev. E 67, 060104(R) (2003). 
[18] A. Toth, G. Schuszter, N. Prabha Das, E. Lantos, D. Horvath, A. De Wit, and F. Brau, Effects of radial injection and solution thickness on the dynamics of confined $A+B \rightarrow C$ chemical fronts, Phys. Chem. Chem. Phys. 22, 10278 (2020).

[19] M. Chalfie, Gfp: Lighting up life, Proc. Natl. Acad. Sci. USA 106, 10073 (2009).

[20] K. D. Piatkevich, S. Bensussen, H. Tseng, S. N. Shroff, V. G. Lopez-Huerta, D. Park, E. E. Jung, O. A. Shemesh, C. Straub, H. J. Gritton, M. F. Romano, E. Costa, B. L. Sabatini, Z. Fu, E. S. Boyden, and X. Han, Population imaging of neural activity in awake behaving mice, Nature (London) 574, 413 (2019).

[21] A. Terskikh et al., "Fluorescent timer": Protein that changes color with time, Science 290, 1585 (2000).

[22] C. V. H. H. Chen, Y. Liu, H. A. Stone, and R. K. Prud'homme, Visualization of surfactant dynamics to and along oil-water interfaces using solvatochromic fluorescent surfactants, Langmuir 34, 10512 (2018).

[23] N. Mingotti and S. S. S. Cardoso, Mixing and reaction in turbulent plumes: The limits of slow and instantaneous chemical kinetics, J. Fluid Mech. 861, 1 (2019).

[24] L. Gálfi and Z. Rácz, Properties of the reaction front in an $A+B \rightarrow C$ type reaction-diffusion process, Phys. Rev. A 38, 3151 (1988).

[25] H. Larralde, M. Araujo, S. Havlin, and H. E. Stanley, Reaction front for $A+B \rightarrow C$ diffusion-reaction systems with initially separated reactants, Phys. Rev. A 46, 855 (1992).

[26] M. Z. Bazant and H. A. Stone, Asymptotics of reaction-diffusion fronts with one static and one diffusing reactant, Phys. D (Amsterdam, Neth.) 147, 95 (2000).

[27] Z. Koza, The long-time behavior of initially separated $A+B \rightarrow 0$ reaction-diffusion systems with arbitrary diffusion constants, J. Stat. Phys. 85, 179 (1996).

[28] H. Taitelbaum, Segregation in reaction-diffusion systems, Phys. A (Amsterdam, Neth.) 200, 155 (1993).

[29] H. Taitelbaum and Z. Koza, Anomalous kinetics of reaction diffusion fronts, Philos. Mag. B 77, 1389 (1998).

[30] B. Budowle, J. L. Leggitt, D. A. Defenbaugh, K. M. Keys, and S. F. Malkiewicz, The presumptive reagent fluorescein for detection of dilute bloodstains and subsequent STR typing of recovered DNA, J. Forensic Sci. 45, 14835J (2000).

[31] C. P. LeBel, H. Ischiropoulos, and S. C. Bondy, Evaluation of the probe 2', 7'- dichlorofluorescin as an indicator of reactive oxygen species formation and oxidative stress, Chem. Res. Toxicol. 5, 227 (1992).

[32] W. M. Haynes, CRC Handbook of Chemistry and Physics (CRC Press, 2014).

[33] E. Guilbert, Ph.D. thesis, Aix-Marseille Université, 2020.

[34] UCON 75-H-90,000, Available from DOW Company, Michigan, USA (2015), https://www.dow.com/enus/pdp.ucon-lubricant-75-h-90000.85853z.html.

[35] A. De Wit, Chemo-hydrodynamic patterns and instabilities, Annu. Rev. Fluid Mech. 52, 531 (2020).

[36] Y. Shi and K. Eckert, A novel Hele-Shaw cell design for the analysis of hydrodynamic instabilities in liquid-liquid systems, Chem. Eng. Sci. 63, 3560 (2008).

[37] G. K. Batchelor, Small-scale variation of convected quantities like temperature in turbulent fluid Part 1. General discussion and the case of small conductivity, J. Fluid Mech. 5, 113 (1959).

[38] H. Taitelbaum, S. Havlin, J. E. Kiefer, B. Trus, and G. H. Weiss, Some properties of the $A+B \rightarrow C$ reaction-diffusion system with initially separated components, J. Stat. Phys. 65, 873 (1991).

[39] M. Sinder and J. Pelleg, Asymptotic properties of a reversible $A+B \leftrightarrow C$ (static) reaction-diffusion process with initially separated reactants, Phys. Rev. E 62, 3340 (2000).

[40] M. Sinder, V. Sokolovsky, and J. Pelleg, Reversible $A \leftrightarrow B$ reaction-diffusion process with initially mixed reactants: Boundary layer function approach, Phys. B (Amsterdam, Neth.) 406, 3042 (2011).

[41] C. H. Gibson and P. A. Libby, On turbulent flows with fast chemical reactions. Part II. The distribution of reactants and products near a reacting surface, Combust. Sci. Technol. 6, 29 (1972).

[42] J. Salvinien and J. J. Moreau, Etude détaillée du mouvement et de la stabilisation du front de précipitation lorsque deux réactifs diffusent l'un vers l'autre dans un gel, J. Chim. Phys. Phys.-Chim. Biol. 55, 300 (1958).

[43] H. S. Carslaw and J. C. Jaeger, Conduction of Heat in Solids (Oxford University Press, London, 1959).

[44] G. I. Taylor, Dispersion of soluble matter in solvent flowing slowly through a tube, Proc. R. Soc. London, Ser. A 219, 186 (1953). 
[45] F. Brau, G. Schuszter, and A. De Wit, Flow Control of $A+B \rightarrow C$ Fronts by Radial Injection, Phys. Rev. Lett. 118, 134101 (2017).

[46] E. Guilbert, B. Metzger, and E. Villermaux, Chemical production on a deforming substrate (unpublished).

[47] E. Guilbert and E. Villermaux, Chemical reactions rectify mixtures composition, Phys. Rev. Fluids 6, L112501 (2021). 